\title{
Color-allowed Bottom Baryon to $s$-wave and $p$-wave Charmed Baryon non-leptonic Decays
}

\section{Chun-Khiang Chua*}

Department of Physics and Center for High Energy Physics Chung Yuan Christian University Chung-Li, Taiwan 320, Republic of China

E-mail: ckchua@cycu.edu.tw

We study color allowed bottom baryon to $s$-wave and $p$-wave charmed baryon non-leptonic decays in this work. The charmed baryons include spin-1/2 and spin-3/2 states. Explicitly, we consider $\Lambda_{b} \rightarrow \Lambda_{c}^{(*, *)} M^{-}, \Xi_{b} \rightarrow \Xi_{c}^{(* *)} M^{-}$and $\Omega_{b} \rightarrow \Omega_{c}^{(*, *)} M^{-}$decays with $M=$ $\pi, K, \rho, K^{*}, a_{1}, D, D_{s}, D^{*}, D_{s}^{*}, \Lambda_{c}^{(* * *)}=\Lambda_{c}, \Lambda_{c}(2595), \Lambda_{c}(2625), \Lambda_{c}(2765), \Lambda_{c}(2940), \Xi_{c}^{(* *)}=\Xi_{c}$, $\Xi_{c}(2790), \Xi_{c}(2815)$ and $\Omega_{c}^{(* * *)}=\Omega_{c}, \Omega_{c}(2770), \Omega_{c}(3050), \Omega_{c}(3090), \Omega_{c}(3120)$. There are six types of transitions, namely, (i) $\mathscr{B}_{b}\left(\overline{\mathbf{3}}_{\mathbf{f}}, 1 / 2^{+}\right)$to $\mathscr{B}_{c}\left(\overline{\mathbf{3}}_{\mathbf{f}}, 1 / 2^{+}\right)$, (ii) $\mathscr{B}_{b}\left(\mathbf{6}_{\mathbf{f}}, 1 / 2^{+}\right)$to $\mathscr{B}_{c}\left(\mathbf{6}_{\mathbf{f}}, 1 / 2^{+}\right)$, (iii) $\mathscr{B}_{b}\left(\mathbf{6}_{\mathbf{f}}, 1 / 2^{+}\right)$to $\mathscr{B}_{c}\left(\mathbf{6}_{\mathbf{f}}, 3 / 2^{+}\right)$, (iv) $\mathscr{B}_{b}\left(\mathbf{6}_{\mathbf{f}}, 1 / 2^{+}\right)$to $\mathscr{B}_{c}\left(\mathbf{6}_{\mathbf{f}}, 3 / 2^{-}\right)$, (v) $\mathscr{B}_{b}\left(\overline{\mathbf{3}}_{\mathbf{f}}, 1 / 2^{+}\right)$to $\mathscr{B}_{c}\left(\overline{\mathbf{3}}_{\mathbf{f}}, 1 / 2^{-}\right)$, and (vi) $\mathscr{B}_{b}\left(\overline{\mathbf{3}_{\mathbf{f}}}, 1 / 2^{+}\right)$to $\mathscr{B}_{c}\left(\mathbf{6}_{\mathbf{f}}, 3 / 2^{-}\right)$transitions. Types (i) to (iii) involve spin $1 / 2$ and $3 / 2 s$-wave charmed baryons, while types (iv) to (vi) involve spin $1 / 2$ and $3 / 2 p$-wave charmed baryons. The light diquarks are spectating in these transitions. The transition form factors are calculated in the light-front quark model approach. All of the form factors in the $1 / 2 \rightarrow 1 / 2$ and $1 / 2 \rightarrow 3 / 2$ transitions are extracted, and they are found to reasonably satisfy the relations obtained in the heavy quark limit, as we are using heavy but finite $m_{b}$ and $m_{c}$. Using naïve factorization, decay rates and up-down asymmetries of the above modes are predicted and can be checked experimentally. The study on these decay modes may shed light on the quantum numbers of $\Lambda_{c}(2765), \Lambda_{c}(2940), \Omega_{c}(3050), \Omega_{c}(3090)$ and $\Omega_{c}(3120)$.

40th International Conference on High Energy physics - ICHEP2020

July 28 - August 6, 2020

Prague, Czech Republic (virtual meeting)

*Speaker. 
In year $2017 \mathrm{LHCb}$ discovered $\Lambda_{c}(2864)$ and five $\Omega_{c}$ states, namely $\Omega_{c}(3000)^{0}, \Omega_{c}(3050)^{0}$, $\Omega_{c}(3066)^{0}, \Omega_{c}(3090)^{0}$ and $\Omega_{c}(3120)^{0}$ [1]. More than one third of charmed baryons have unspecified quantum numbers [2]. Those include $\Lambda_{c}(2765)^{+}, \Sigma_{c}(2800)^{++,+, 0}, \Xi_{c}(2930)^{0}, \Xi_{c}(2970)^{+, 0}$, $\Xi_{c}(3055)^{+}, \Xi_{c}(3080)^{+, 0}, \Xi_{c}(3123)^{+}$and the above mentioned five $\Omega_{c}$ states. Various suggestions on the quantum numbers of the newly discovered $\Omega_{c}$ states were put forward after the discovery, see, for example, [3] and references therein. Note that even for the one with specified quantum number in PDG, it is still room for different assignments. Two possible quantum numbers of $\Lambda_{c}(2940)^{+}$were proposed. LHCb and PDG preferred the $\frac{3}{2}^{-}$quantum number, but it is not certain $[1,2]$, while a $\frac{1}{2}^{-}$state was advocated by the authors of ref. [3]. It is timely, of great interest and importance to identify the quantum numbers of these states and to study their properties.

The study of bottom baryon to charmed baryon weak decays may shed light on the quantum numbers of charmed baryons. Up to now only several color allowed $\Lambda_{b} \rightarrow \Lambda_{c} P$ decay rates were measured. These include the rates of $\Lambda_{b} \rightarrow \Lambda_{c} \pi^{-}, \Lambda_{c} K^{-}, \Lambda_{c} D^{-}$and $\Lambda_{c} D_{s}^{-}$decays, which were reported by LHCb several years ago [4]. It is not unrealistic to expect that further progress on the experimental side, either from LHCb, from Belle II or from elsewhere, will occur soon. In [5] we studied the color allowed $\Lambda_{b}^{0} \rightarrow \Lambda_{c}^{(*, * *)} M, \Xi_{b} \rightarrow \Xi_{c}^{(* *)} M$ and $\Omega_{b} \rightarrow \Omega_{c}^{(*)} M$ decays with $M=\pi, K, \rho, K^{*}, a_{1}, D, D_{s}, D^{*}, D_{s}^{*}$, and $\Lambda_{c}^{(*, * *)}=\Lambda_{c}, \Lambda_{c}(2595), \Lambda_{c}(2765), \Lambda_{c}(2940), \Xi_{c}^{(* *)}=\Xi_{c}$, $\Xi_{c}(2790)$ and $\Omega_{c}^{(*)}=\Omega_{c}, \Omega_{c}(3090)$, which are spin-1/2 charm baryons. In [6] we extend the study to $s$-wave and $p$-wave charmed baryons up to spin-3/2 states, these include $\Lambda_{c}^{(*, * *)}=\Lambda_{c}, \Lambda_{c}(2595)$, $\Lambda_{c}(2625), \Lambda_{c}(2765), \Lambda_{c}(2940)(\operatorname{spin}-1 / 2), \Lambda_{c}(2940)(\operatorname{spin}-3 / 2), \Xi_{c}^{(*)}=\Xi_{c}, \Xi_{c}(2790), \Xi_{c}(2815)$ and $\Omega_{c}^{(*, *)}=\Omega_{c}, \Omega_{c}(2770), \Omega_{c}(3050), \Omega_{c}(3090), \Omega_{c}(3120)$. This proceeding is based on these two works $[5,6]$.

We consider the color allowed non-leptonic two body weak decays, where the light quarks are spectating in the processes. These transitions are straightforward and easier to study. There are six types of $\mathscr{B}_{b} \rightarrow \mathscr{B}_{c}$ transitions, namely, (i) $\mathscr{B}_{b}\left(\overline{\mathbf{3}}_{\mathbf{f}}, 1 / 2^{+}\right)$to $\mathscr{B}_{c}\left(\overline{\mathbf{3}}_{\mathbf{f}}, 1 / 2^{+}\right)$, (ii) $\mathscr{B}_{b}\left(\mathbf{6}_{\mathbf{f}}, 1 / 2^{+}\right)$ to $\mathscr{B}_{c}\left(\mathbf{6}_{\mathbf{f}}, 1 / 2^{+}\right)$, (iii) $\mathscr{B}_{b}\left(\mathbf{6}_{\mathbf{f}}, 1 / 2^{+}\right)$to $\mathscr{B}_{c}\left(\mathbf{6}_{\mathbf{f}}, 3 / 2^{+}\right)$, (iv) $\mathscr{B}_{b}\left(\mathbf{6}_{\mathbf{f}}, 1 / 2^{+}\right)$to $\mathscr{B}_{c}\left(\mathbf{6}_{\mathbf{f}}, 3 / 2^{-}\right),(\mathrm{v})$ $\mathscr{B}_{b}\left(\overline{\mathbf{3}}_{\mathbf{f}}, 1 / 2^{+}\right)$to $\mathscr{B}_{c}\left(\overline{\mathbf{3}}_{\mathbf{f}}, 1 / 2^{-}\right)$, and (vi) $\mathscr{B}_{b}\left(\overline{\mathbf{3}}_{\mathbf{f}}, 1 / 2^{+}\right)$to $\mathscr{B}_{c}\left(\overline{\mathbf{3}}_{\mathbf{f}}, 3 / 2^{-}\right)$transitions. Types (i) to (iii) involve spin $1 / 2$ and $3 / 2 s$-wave charmed baryons, while types (iv) to (vi) involve spin $1 / 2$ and $3 / 2 p$-wave charmed baryons. Since there are two possible quantum number assignments for $\Lambda_{c}(2940)$, namely a radial excited $p$-wave spin- $1 / 2$ or a spin-3/2 state, it will be useful to consider both possibilities and compare the predictions on rates and so on. The transition form factors are calculated in the light-front quark model approach. All of the form factors in the $1 / 2 \rightarrow 1 / 2$ and $1 / 2 \rightarrow 3 / 2$ transitions are extracted, and they are found to reasonably satisfy the relations obtained in the heavy quark limit, as we are using heavy but finite $m_{b}$ and $m_{c}$.

The predicted branching ratios for $\mathscr{B}_{b} \rightarrow \mathscr{B}_{c} P$ and $\mathscr{B}_{b} \rightarrow \mathscr{B}_{c} V, \mathscr{B}_{c} A$ decays using naïve factorization, are summarized in Tables 1 and 2, respectively. In Tables 3 and 4, we show the prediction of up-down asymmetries for $\mathscr{B}_{b} \rightarrow \mathscr{B}_{c} M$ decays with $M=P, V, A$. As shown in Tables 5 and 6, overall speaking our results of rates and asymmetries agree well within errors with existing data and almost all of the results obtained in other works. For example, our results on $\Lambda_{b} \rightarrow$ $\Lambda_{c} \pi^{-}, \Lambda_{c} K^{-}, \Lambda_{c} D^{-}, \Lambda_{c} D_{s}^{-}$rates can be compared to the following experimental results, $\operatorname{Br}\left(\Lambda_{b} \rightarrow\right.$ $\left.\Lambda_{c} \pi^{-}, \Lambda_{c} K^{-}, \Lambda_{c} D^{-}, \Lambda_{c} D_{s}^{-}\right)=(4.9 \pm 0.4,0.359 \pm 0.030,0.46 \pm 0.06,11.0 \pm 1.0) \times 10^{-3}$ [2]. The signs of asymmetries $\alpha$ are mostly negative, while those in $\Omega_{b} \rightarrow \Omega_{c} M, \Omega_{b} \rightarrow \Omega_{c}(3050) M$ and 
Table 1: Branching ratios (in the unit of $10^{-3}$ ) of $\mathscr{B}_{b} \rightarrow \mathscr{B}_{c} P$ decays. Note that the asterisks denote the transitions where the final state charmed baryons are radial excited.

\begin{tabular}{|c|c|c|c|c|c|}
\hline Transition type & Mode & $P=\pi^{-}$ & $P=K^{-}$ & $P=D^{-}$ & $P=D_{s}^{-}$ \\
\hline (i) $\left(\frac{1}{2}^{+} \rightarrow \frac{1}{2}^{+}\right)$ & $\operatorname{Br}\left(\Lambda_{b} \rightarrow \Lambda_{c} P\right)$ & $4.16_{-1.73}^{+2.43}$ & $0.31_{-0.13}^{+0.18}$ & $0.47_{-0.21}^{+0.30}$ & $11.92_{-5.28}^{+7.69}$ \\
\hline (v) $\left(\frac{1}{2}^{+} \rightarrow \frac{1}{2}^{-}\right)$ & $\operatorname{Br}\left[\Lambda_{b} \rightarrow \Lambda_{c}(2595) P\right]$ & $1.09_{-0.51}^{+0.76}$ & $0.08_{-0.04}^{+0.06}$ & $0.07_{-0.04}^{+0.07}$ & $1.72_{-1.01}^{+1.71}$ \\
\hline (vi) $\left(\frac{1}{2}^{+} \rightarrow \frac{3}{2}\right.$ & $\operatorname{Br}\left[\Lambda_{b} \rightarrow \Lambda_{c}(2625) P\right]$ & $2.40_{-1.82}^{+4.09}$ & $0.17_{-0.13}^{+0.30}$ & $0.13_{-0.10}^{+0.22}$ & $2.88_{-2.16}^{+4.92}$ \\
\hline$(\mathrm{i})^{*}\left(\frac{1}{2}^{+}-\right.$ & $\operatorname{Br}\left[\Lambda_{b} \rightarrow \Lambda_{c}(2765) P\right]$ & $1.70_{-0.52}^{+0.69}$ & $0.13_{-0.04}^{+0.05}$ & $0.15_{-0.05}^{+0.07}$ & $\begin{array}{l}3.54_{-1.24}^{+1.73} \\
-\end{array}$ \\
\hline$(\mathrm{v})^{*}($ & $\operatorname{Br}\left[\Lambda_{b} \rightarrow \Lambda_{c}(2940) P\right]$ & $0.68_{-0.21}^{+0.21}$ & $0.05_{-0.02}^{+0.02}$ & $0.04_{-0.02}^{+0.02}$ & $0.87_{-0.38}^{+0.46}$ \\
\hline$(\mathrm{vi})^{*}\left(\frac{1}{2}^{+}\right.$ & $\operatorname{Br}\left[\Lambda_{b} \rightarrow \Lambda_{c}(2940) P\right]$ & $1.00_{-0.83}^{+2.00}$ & $0.07_{-0.06}^{+0.14}$ & $0.07_{-0.05}^{+0.10}$ & $1.69_{-1.23}^{+2.30}$ \\
\hline & $\operatorname{Br}\left(\Xi_{b}^{-} \rightarrow \Xi_{c}^{0} P\right)$ & $3.88_{-1.69}^{+2.43}$ & $0.29_{-0.13}^{+0.18}$ & $0.45_{-0.21}^{+0.31}$ & $11.54_{-5.34}^{+7.98}$ \\
\hline (i) $\left(\frac{1}{2}\right.$ & $\operatorname{Br}\left(\Xi_{b}^{0} \rightarrow \Xi_{c}^{+} P\right)$ & $3.66_{-1.59}^{+2.29}$ & $0.28_{-0.12}^{+0.17}$ & $0.43_{-0.20}^{+0.29}$ & $10.87_{-5.03}^{+7.51}$ \\
\hline (v) $\left(\frac{1}{2}^{+} \rightarrow \frac{1}{2}^{-}\right)$ & $\operatorname{Br}\left[\Xi_{b}^{-} \rightarrow \Xi_{c}^{0}(2790) P\right]$ & $1.03_{-0.48}^{+0.79}$ & $0.08_{-0.04}^{+0.06}$ & $0.07_{-0.04}^{+0.08}$ & $1.70_{-0.99}^{+1.88}$ \\
\hline (v) $\left(\frac{1}{2}^{+} \rightarrow \frac{1}{2}^{-}\right)$ & $\operatorname{Br}\left[\Xi_{b}^{0} \rightarrow \Xi_{c}^{-}(2790) P\right]$ & $0.97_{-0.45}^{+0.74}$ & $0.07_{-0.03}^{+0.06}$ & $0.07_{-0.04}^{+0.07}$ & $1.60_{-0.93}^{+1.76}$ \\
\hline (vi) $\left(\frac{1}{2}^{+} \rightarrow \frac{3}{2}^{-}\right)$ & $\operatorname{Br}\left[\Xi_{b}^{-} \rightarrow \Xi_{c}^{0}(2815) P\right]$ & $3.53_{-2.80}^{+6.46}$ & $0.26_{-0.20}^{+0.47}$ & $0.20_{-0.15}^{+0.35}$ & $4.65_{-3.48}^{+8.08}$ \\
\hline (vi) $\left(\frac{1}{2}^{+} \rightarrow \frac{3}{2}^{-}\right)$ & $\operatorname{Br}\left[\Xi_{b}^{0} \rightarrow \Xi_{c}^{+}(2815) P\right]$ & $3.32_{-2.63}^{+6.08}$ & $0.24_{-0.19}^{+0.44}$ & $0.19_{-0.14}^{+0.33}$ & $4.34_{-3.25}^{+7.54}$ \\
\hline (ii) $\left(\frac{1}{2}^{+} \rightarrow \frac{1}{2}^{+}\right)$ & $\operatorname{Br}\left(\Omega_{b} \rightarrow \Omega_{c} P\right)$ & $1.10_{-0.55}^{+0.85}$ & $0.08_{-0.04}^{+0.07}$ & $0.15_{-0.08}^{+0.14}$ & $4.03_{-2.21}^{+3.72}$ \\
\hline (iii) $\left(\frac{1}{2}^{+} \rightarrow \frac{3}{2}^{+}\right)$ & $\operatorname{Br}\left[\Omega_{b} \rightarrow \Omega_{c}(2770) P\right]$ & $1.37_{-1.19}^{+3.01}$ & $0.11_{-0.09}^{+0.23}$ & $0.28_{-0.20}^{+0.38}$ & $7.46_{-5.04}^{+9.63}$ \\
\hline (iv) $\left(\frac{1}{2}^{+} \rightarrow \frac{3}{2}^{-}\right)$ & $\operatorname{Br}\left[\Omega_{b} \rightarrow \Omega_{c}(3050) P\right]$ & $3.40_{-2.25}^{+4.45}$ & $0.24_{-0.16}^{+0.31}$ & $0.09_{-0.07}^{+0.15}$ & $1.78_{-1.38}^{+3.16}$ \\
\hline$(\mathrm{ii})^{*}\left(\frac{1}{2}^{+} \rightarrow \frac{1}{2}^{+}\right)$ & $\operatorname{Br}\left[\Omega_{b} \rightarrow \Omega_{c}(3090) P\right]$ & $0.85_{-0.35}^{+0.50}$ & $0.06_{-0.03}^{+0.04}$ & $0.10_{-0.05}^{+0.07}$ & $2.43_{-1.15}^{+1.79}$ \\
\hline$(\mathrm{iii})^{*}\left(\frac{1}{2}^{+} \rightarrow \frac{3}{2}^{+}\right)$ & $\operatorname{Br}\left[\Omega_{b} \rightarrow \Omega_{c}(3120) P\right]$ & $0.96_{-0.52}^{+0.95}$ & $0.07_{-0.04}^{+0.07}$ & $0.10_{-0.05}^{+0.08}$ & $2.37_{-1.10}^{+1.81}$ \\
\hline
\end{tabular}

Table 2: Same as Table 1 but for $\mathscr{B}_{b} \rightarrow \mathscr{B}_{c} V$ and $B_{b} \rightarrow \mathscr{B}_{c} A$ decays.

\begin{tabular}{|c|c|c|c|c|c|c|}
\hline Type & Mode & $M=\rho^{-}$ & $M=K^{*-}$ & $M=D^{*-}$ & $M=D_{s}^{*-}$ & $M=a_{1}^{-}$ \\
\hline (i) & $\operatorname{Br}\left(\Lambda_{b} \rightarrow \Lambda_{c} M\right)$ & $12.28_{-5.11}^{+7.19}$ & $0.63_{-0.26}^{+0.37}$ & $0.84_{-0.36}^{+0.51}$ & $17.49_{-7.48}^{+10.60}$ & $11.91_{-4.97}^{+6.98}$ \\
\hline (v) & $\operatorname{Br}\left[\Lambda_{b} \rightarrow \Lambda_{c}(2595) M\right]$ & $2.99_{-1.44}^{+2.20}$ & $0.15_{-0.07}^{+0.11}$ & $0.12_{-0.07}^{+0.11}$ & $2.28_{-1.29}^{+2.21}$ & $2.57_{-1.29}^{+2.01}$ \\
\hline (vi) & $\operatorname{Br}\left[\Lambda_{b} \rightarrow \Lambda_{c}(2625) M\right]$ & $4.38_{-3.17}^{+6.78}$ & $0.22_{-0.16}^{+0.33}$ & $0.13_{-0.08}^{+0.017}$ & $2.41_{-1.52}^{+2.98}$ & $3.50_{-2.45}^{+5.11}$ \\
\hline$(i)^{*}$ & $\operatorname{Br}\left[\Lambda_{b} \rightarrow \Lambda_{c}(2765) M\right]$ & $4.84_{-1.50}^{+2.01}$ & $0.25_{-0.08}^{+0.10}$ & $0.26_{-0.09}^{+0.12}$ & $5.29_{-1.84}^{+2.54}$ & $4.45_{-1.42}^{+1.91}$ \\
\hline$(\mathrm{v})^{*}$ & $\operatorname{Br}\left[\Lambda_{b} \rightarrow \Lambda_{c}(2940) M\right]$ & $1.85_{-0.60}^{+0.63}$ & $0.09_{-0.03}^{+0.03}$ & $0.06_{-0.03}^{+0.03}$ & $1.16_{-0.48}^{+0.62}$ & $1.57_{-0.54}^{+0.59}$ \\
\hline$(\mathrm{vi})^{*}$ & $\operatorname{Br}\left[\Lambda_{b} \rightarrow \Lambda_{c}(2940) M\right]$ & $1.93_{-1.43}^{+3.19}$ & $0.10_{-0.07}^{+0.15}$ & $0.06_{-0.03}^{+0.06}$ & $1.11_{-0.62}^{+1.07}$ & $1.58_{-1.08}^{+2.26}$ \\
\hline (i) & $\operatorname{Br}\left(\Xi_{b}^{-} \rightarrow \Xi_{c}^{0} M\right)$ & $11.56_{-5.04}^{+7.25}$ & $0.60_{-0.26}^{+0.37}$ & $0.82_{-0.37}^{+0.53}$ & $17.26_{-7.70}^{+11.2}$ & $11.37_{-4.97}^{+7.14}$ \\
\hline (i) & $\operatorname{Br}\left(\Xi_{b}^{0} \rightarrow \Xi_{c}^{+} M\right)$ & $10.88_{-4.74}^{+6.83}$ & $0.56_{-0.24}^{+0.35}$ & $0.77_{-0.35}^{+0.50}$ & $16.24_{-7.25}^{+10.54}$ & $10.70_{-4.67}^{+6.72}$ \\
\hline (v) & $\operatorname{Br}\left[\Xi_{b}^{-} \rightarrow \Xi_{c}^{0}(2790) M\right]$ & $2.86_{-1.36}^{+2.28}$ & $0.14_{-0.07}^{+0.12}$ & $0.12_{-0.06}^{+0.12}$ & $2.25_{-1.26}^{+2.33}$ & $2.48_{-1.23}^{+2.10}$ \\
\hline (v) & $\operatorname{Br}\left[\Xi_{b}^{0} \rightarrow \Xi_{c}^{+}(2790) M\right]$ & $2.69_{-1.28}^{+2.15}$ & $0.13_{-0.06}^{+0.11}$ & $0.11_{-0.06}^{+0.11}$ & $2.11_{-1.19}^{+2.19}$ & $2.33_{-1.16}^{+1.98}$ \\
\hline (vi) & $\operatorname{Br}\left[\Xi_{b}^{-} \rightarrow \Xi_{c}^{0}(2815) M\right]$ & $6.49_{-4.84}^{+10.58}$ & $0.32_{-0.24}^{+0.51}$ & $0.20_{-0.13}^{+0.26}$ & $3.74_{-2.32}^{+4.58}$ & $5.24_{-3.72}^{+7.92}$ \\
\hline (vi) & $\operatorname{Br}\left[\Xi_{b}^{0} \rightarrow \Xi_{c}^{+}(2815) M\right]$ & $6.10_{-4.55}^{+9.95}$ & $0.30_{-0.22}^{+0.48}$ & $0.19_{-0.12}^{+0.24}$ & $3.51_{-2.18}^{+4.30}$ & $4.92_{-3.50}^{+7.45}$ \\
\hline (ii) & $\operatorname{Br}\left(\Omega_{b} \rightarrow \Omega_{c} M\right)$ & $3.07_{-1.53}^{+2.41}$ & $0.16_{-0.08}^{+0.12}$ & $0.16_{-0.08}^{+0.13}$ & $3.18_{-1.61}^{+2.69}$ & $2.76_{-1.37}^{+2.20}$ \\
\hline (iii) & $\operatorname{Br}\left[\Omega_{b} \rightarrow \Omega_{c}(2770) M\right]$ & $2.37_{-1.85}^{+4.68}$ & $0.13_{-0.10}^{+0.24}$ & $0.28_{-0.16}^{+0.30}$ & $6.20_{-3.49}^{+6.19}$ & $2.78_{-1.93}^{+4.35}$ \\
\hline (iv) & $\operatorname{Br}\left[\Omega_{b} \rightarrow \Omega_{c}(3050) M\right]$ & $4.09_{-2.71}^{+5.62}$ & $0.20_{-0.13}^{+0.10}$ & $0.08_{-0.05}^{+0.10}$ & $1.43_{-0.88}^{+1.78}$ & $\begin{array}{l}2.84_{-1.86}^{+3.88} \\
-\end{array}$ \\
\hline$(\text { ii })^{*}$ & $\operatorname{Br}\left[\Omega_{b} \rightarrow \Omega_{c}(3090) M\right]$ & $2.29_{-0.94}^{+1.36}$ & $0.11_{-0.05}^{+0.07}$ & $0.09_{-0.04}^{+0.06}$ & $1.69_{-0.71}^{+1.06}$ & $1.92_{-0.79}^{+1.15}$ \\
\hline$(\text { iii) })^{*}$ & $\operatorname{Br}\left[\Omega_{b} \rightarrow \Omega_{c}(3120) M\right]$ & $1.50_{-0.76}^{+1.37}$ & $0.08_{-0.04}^{+0.07}$ & $0.11_{-0.04}^{+0.07}$ & $2.37_{-0.88}^{+1.33}$ & $1.55_{-0.71}^{+1.21}$ \\
\hline
\end{tabular}


Table 3: The predicted up-down asymmetries (in the unit of $\%$ ) of $\mathscr{B}_{b} \rightarrow \mathscr{B}_{c} P$ decays. Note that the asterisks denote the transitions where the final state charmed baryons are radial excited.

\begin{tabular}{|c|c|c|c|c|c|}
\hline Type & Mode & $P=\pi^{-}$ & $P=K^{-}$ & $P=D^{-}$ & $P=D_{s}^{-}$ \\
\hline (i) $\left(\frac{1}{2}^{+} \rightarrow \frac{1}{2}^{+}\right)$ & $\alpha\left(\Lambda_{b} \rightarrow \Lambda_{c} P\right)$ & $-99.99_{-0.00}^{+4.70}$ & $-99.97_{-0.01}^{+5.02}$ & $-99.45_{-0.55}^{+7.94}$ & $-99.19_{-0.81}^{+8.59}$ \\
\hline (v) $\left(\frac{1}{2}^{+} \rightarrow \frac{1}{2}\right.$ & $\alpha\left[\Lambda_{b} \rightarrow \Lambda_{c}(2595) P\right]$ & $-98.33_{-1.67}^{+12.89}$ & $-98.12_{-1.88}^{+13.51}$ & $-88.05_{-11.95}^{+26.57}$ & $-86.49_{-13.51}^{+27.83}$ \\
\hline (vi) $\left(\frac{1}{2}^{+} \rightarrow \frac{3}{2}^{-}\right)$ & $\alpha\left[\Lambda_{b} \rightarrow \Lambda_{c}(2625) P\right]$ & $-97.76_{-2.24}^{+39.39}$ & $-97.64_{-2.36}^{+39.37}$ & $-97.44_{-2.56}^{+37.86}$ & $-97.07_{-2.93}^{+38.04}$ \\
\hline$(\mathrm{i})^{*}\left(\frac{1}{2}^{+} \rightarrow \frac{1}{2}^{+}\right)$ & $\alpha\left[\Lambda_{b} \rightarrow \Lambda_{c}(2765) P\right]$ & $-99.93_{-0.02}^{+1.65}$ & $-99.87_{-0.11}^{+1.87}$ & $-98.03_{-1.97}^{+5.04}$ & $-97.23_{-2.70}^{+5.70}$ \\
\hline$(\mathrm{v})^{*}\left(\frac{1}{2}^{+}\right.$ & $\alpha\left[\Lambda_{b} \rightarrow \Lambda_{c}(2940) P\right]$ & $-98.32_{-1}^{+2}$ & $-98.10_{-1.64}^{+3.11}$ & $-86.24_{-9.26}^{+11.11}$ & $-84.04_{-10.66}^{+12.19}$ \\
\hline$(\mathrm{vi})^{*}\left(\frac{1}{2}^{+} \rightarrow \frac{3}{2}^{-}\right.$ & $\alpha\left[\Lambda_{b} \rightarrow \Lambda_{c}(2940) P\right]$ & $-99.41_{-0.59}^{+65.88}$ & $-99.06_{-0.94}^{+61.14}$ & $-89.25_{-10.75}^{+31.59}$ & $-86.81_{-13.19}^{+30.72}$ \\
\hline (i) $\left(\frac{1}{2}^{+} \rightarrow \frac{1}{2}^{+}\right)$ & $\alpha\left(\Xi_{b}^{-} \rightarrow \Xi_{c}^{0} P\right)$ & $-99.98_{-0.00}^{+5.73}$ & $-99.96_{-0.00}^{+6.10}$ & $-99.29_{-0.71}^{+9.61}$ & $-98.99_{-1.01}^{+10.34}$ \\
\hline (i) $\left(\frac{1}{2}^{+} \rightarrow \frac{1}{2}^{+}\right)$ & $\alpha\left(\Xi_{b}^{0} \rightarrow \Xi_{c}^{+} P\right)$ & $-99.98_{-0.00}^{+5.73}$ & $-99.96_{-0.00}^{+6.10}$ & $-99.29_{-0.71}^{+9.61}$ & $-98.99_{-1.01}^{+10.34}$ \\
\hline (v) $\left(\frac{1}{2}^{+} \rightarrow \frac{1}{2}^{-}\right)$ & $\alpha\left[\Xi_{b}^{-} \rightarrow \Xi_{c}^{0}(2790) P\right]$ & $-98.13_{-1.87}^{+14.56}$ & $-97.88_{-2.11}^{+15.26}$ & $-86.62_{-13.38}^{+28.66}$ & $-84.85_{-15.15}^{+29.84}$ \\
\hline (v) $\left(\frac{1}{2}^{+} \rightarrow \frac{1}{2}^{-}\right.$ & $\alpha\left[\Xi_{b}^{0} \rightarrow \Xi_{c}^{-}(2790) P\right]$ & $-98.13_{-1.87}^{+14.56}$ & $-97.88_{-2.11}^{+15.26}$ & $-86.60_{-13.40}^{+28.67}$ & $-84.83_{-15.16}^{+29.85}$ \\
\hline (vi) $\left(\frac{1}{2}^{+} \rightarrow \frac{3}{2}^{-}\right)$ & $\alpha\left[\Xi_{b}^{-} \rightarrow \Xi_{c}^{0}(2815) P\right]$ & $-97.63_{-2.37}^{+42.32}$ & $-97.48_{-2.52}^{+42.09}$ & $-96.48_{-3.52}^{+38.46}$ & $-95.89_{-4.41}^{+38.40}$ \\
\hline (vi) $\left(\frac{1}{2}^{+} \rightarrow \frac{3}{2}^{-}\right)$ & $\alpha\left[\Xi_{b}^{0} \rightarrow \Xi_{c}^{+}(2815) P\right]$ & $-97.70_{-2.30}^{+42.27}$ & $-97.56_{-2.44}^{+42.03}$ & $-96.71_{-3.29}^{+38.31}$ & $-96.16_{-3.84}^{+38.26}$ \\
\hline & $\alpha\left(\Omega_{b} \rightarrow \Omega_{c} P\right)$ & $59.94_{-18.76}^{+21.34}$ & $59.39_{-18.70}^{+21.45}$ & $56.04_{-19.29}^{+23.79}$ & $55.16_{-19.18}^{+23.98}$ \\
\hline (iii) $\left(\frac{1}{2}^{+} \rightarrow \frac{3}{2}^{+}\right)$ & $\alpha\left[\Omega_{b} \rightarrow \Omega_{c}(2770) P\right]$ & $2.60_{-102.23}^{+97.40}$ & $1.17_{-100.15}^{+98.43}$ & $-11.02_{-59.25}^{+55.88}$ & $-11.70_{-55.10}^{+50.63}$ \\
\hline (iv) $\left(\frac{1}{2}^{+} \rightarrow \frac{3}{2}^{-}\right)$ & $\alpha\left[\Omega_{b} \rightarrow \Omega_{c}(3050) P\right]$ & $18.07_{-41.45}^{+52.52}$ & $17.73_{-42.50}^{+53.31}$ & $9.16_{-71.89}^{+75.06}$ & $7.09_{-76.03}^{+78.45}$ \\
\hline$(\mathrm{ii})^{*}\left(\frac{1}{2}^{+} \rightarrow \frac{1}{2}^{+}\right)$ & $\alpha\left[\Omega_{b} \rightarrow \Omega_{c}(3090) P\right]$ & $59.75_{-13.17}^{+14.13}$ & $59.15_{-13.16}^{+14.21}$ & $54.01_{-13.95}^{+16.49}$ & $52.73_{-13.86}^{+16.61}$ \\
\hline$(\mathrm{iii})^{*}\left(\frac{1}{2}^{+} \rightarrow \frac{3}{2}^{+}\right)$ & $\alpha\left[\Omega_{b} \rightarrow \Omega_{c}(3120) P\right]$ & $4.58_{-41.22}^{+42.35}$ & $3.81_{-40.26}^{+41.17}$ & $-3.74_{-24.05}^{+22.18}$ & $-4.20_{-22.52}^{+20.50}$ \\
\hline
\end{tabular}

Table 4: Same as Table 3 but for $B_{b} \rightarrow \mathscr{B}_{c} V$ and $B_{b} \rightarrow \mathscr{B}_{c} A$ decays.

\begin{tabular}{|c|c|c|c|c|c|c|}
\hline Type & Mode & $M=\rho^{-}$ & $M=K$ & $M=D^{*-}$ & $M=D_{s}^{*-}$ & $M=a_{1}^{-}$ \\
\hline (i) & $\alpha\left(\Lambda_{b} \rightarrow \Lambda_{c} M\right)$ & $-86.96_{-0.87}^{+5.60}$ & $-82.96_{-1}^{+6 .}$ & $-36.85_{-4.88}^{+7.08}$ & $-32.69_{-5.05}^{+6.82}$ & $-70.00_{-2.30}^{+7.00}$ \\
\hline (v) & $\alpha\left[\Lambda_{b} \rightarrow \Lambda_{c}(2595) M\right]$ & $-85.6_{-3.85}^{+12.41}$ & $-81.65_{-4.46}^{+12.21}$ & $-33.47_{-11.40}^{+15.76}$ & $-28.68_{-11.88}^{+15.97}$ & $-68.66_{-6.19}^{+11.32}$ \\
\hline (vi) & $\alpha\left[\Lambda_{b} \rightarrow \Lambda_{c}(2625) M\right]$ & $-91.48_{-3.89}^{+42.04}$ & $-89.01_{-4.91}^{+41.55}$ & $-53.92_{-18.71}^{+34.18}$ & $-49.80_{-20.08}^{+33.31}$ & $-80.52_{-8.58}^{+39.81}$ \\
\hline$(i)^{*}$ & $\alpha\left[\Lambda_{b} \rightarrow \Lambda_{c}(2765) M\right]$ & $-86.15_{-1.04}^{+2.71}$ & $-81.89_{-1.47}^{+3.24}$ & $-31.54_{-4.91}^{+5.31}$ & $-26.87_{-4.82}^{+5.07}$ & $-67.99_{-2}^{+4}$ \\
\hline$(\mathrm{v})^{*}$ & $\alpha\left[\Lambda_{b} \rightarrow \Lambda_{c}(2940) M\right]$ & $-84.01_{-3.07}^{+4.44}$ & $-79.56_{-3.51}^{+4.84}$ & $-24.77_{-9.27}^{+10.24}$ & $-19.04_{-9.71}^{+10.34}$ & $-64.97_{-4}^{+c}$ \\
\hline$(v i)^{*}$ & $\alpha\left[\Lambda_{b} \rightarrow \Lambda_{c}(2940) M\right]$ & $-87.25_{-7.21}^{+66.15}$ & $-84.14_{-8.45}^{+63.92}$ & $-48.02_{-18.07}^{+36.43}$ & $-45.28_{-18.13}^{+33.21}$ & $-73.90_{-12.00}^{+56.57}$ \\
\hline (i) & $\alpha\left(\Xi_{b}^{-} \rightarrow \Xi_{c}^{0} M\right)$ & $-86.61_{-1.09}^{+6.57}$ & $-82.52_{-1.38}^{+7.04}$ & $-36.00_{-5.51}^{+8.09}$ & $5_{-5.73}^{+7.78}$ & $-69.34_{-2.69}^{+8.13}$ \\
\hline (i) & $\alpha\left(\Xi_{b}^{0} \rightarrow \Xi_{c}^{+} M\right)$ & -86.61 & $-82.52_{-1.38}^{+7.04}$ & $-35.98_{-5.51}^{+8.08}$ & $-31.84_{-5.72}^{+7.78}$ & $-69.33_{-269}^{+8.13}$ \\
\hline (v) & $\alpha\left[\Xi_{b}^{-} \rightarrow \Xi_{c}^{0}(2790) M\right]$ & $-85.14_{-}^{+}$ & $-81.11_{-4.77}^{+13.40}$ & $-32.40_{-11.43}^{+16.14}$ & -27.57 & $-67.92_{-6.55}^{+12.18}$ \\
\hline (v) & $\alpha\left[\Xi_{b}^{0} \rightarrow \Xi_{c}^{+}(2790) M\right]$ & $-85.13_{-4.15}^{+13.71}$ & $-81.09_{-4.78}^{+13.40}$ & $-32.35_{-11.43}^{+16.14}$ & $-27.52_{-11.88}^{+16.30}$ & $-67.90_{-6.55}^{+12.17}$ \\
\hline (vi) & $\alpha\left[\Xi_{b}^{-} \rightarrow \Xi_{c}^{0}(2815) M\right]$ & $-91.55_{-4.08}^{+46.65}$ & $-89.08_{-5.17}^{+45.9}$ & $-55.07_{-18.5}^{+35.28}$ & $\begin{array}{r}-51.32_{-1977}^{+34.12} \\
-72\end{array}$ & $-80.63_{-8.94}^{+43.30}$ \\
\hline (vi) & $\alpha\left[\Xi_{b}^{0} \rightarrow \Xi_{c}^{+}(2815) M\right]$ & $-91.48_{-4.15}^{+4.84}$ & $-89.00_{-5.27}^{+46.09}$ & $\begin{array}{r}-54.83_{-18.69}^{+35.37} \\
\end{array}$ & $\begin{array}{r}-51.06_{-20.08}^{+34.18} \\
\end{array}$ & $-80.52_{-9.07}^{+43.48}$ \\
\hline (i) & $\alpha\left(\Omega_{b} \rightarrow \Omega_{c} M\right)$ & $61.63_{-19.83}^{+22.03}$ & $62.20_{-20.19}^{+22.22}$ & $72.15^{+}$ & $73.53_{-26.05}^{+2.54}$ & $64.27_{-21.45}^{+22.74}$ \\
\hline (iii) & $\alpha\left[\Omega_{b} \rightarrow \Omega_{c}(2770\right.$ & -5.22 & $-7.51_{-85}^{+78}$ & $-22.24_{-51.42}^{+45.94}$ & $-22.62_{-48.63}^{+42.82}$ & $-13.64_{-7}^{+6}$ \\
\hline (iv) & $\alpha\left[\Omega_{b} \rightarrow \Omega_{c}(30\right.$ & 24.95 & 24.72 & $13.22_{-62}^{+49}$ & $10.39_{-6}^{+4}$ & $23.53_{-}^{+}$ \\
\hline (ii) & {$\left[\Omega_{b} \rightarrow \Omega_{c}(3090) M\right]$} & & $62.10_{-1}^{+1}$ & $73.1_{-17.68}^{+15.51}$ & $74.63_{-17.81}^{+14.95}$ & $64.31_{-15.17}^{+15.63}$ \\
\hline (iii) $^{*}$ & $\alpha\left[\Omega_{b} \rightarrow \Omega_{c}(3120) M\right]$ & $1.15_{-4}^{+4}$ & $-0.31_{-46.29}^{+41.58}$ & $-11.06_{-24.74}^{+21.35}$ & $-11.43_{-22.92}^{+19.79}$ & $-4.44_{-39.57}^{+33.80}$ \\
\hline
\end{tabular}


Table 5: Comparisons of data and theoretical results on the branching ratios (in the unit of $10^{-3}$ ) of $\Lambda_{b} \rightarrow \Lambda_{c} M, \Xi_{b} \rightarrow \Xi_{c} M, \Omega_{b} \rightarrow \Omega_{c} M$ and $\Omega_{b} \rightarrow \Omega_{c}(2770) M$ decays.

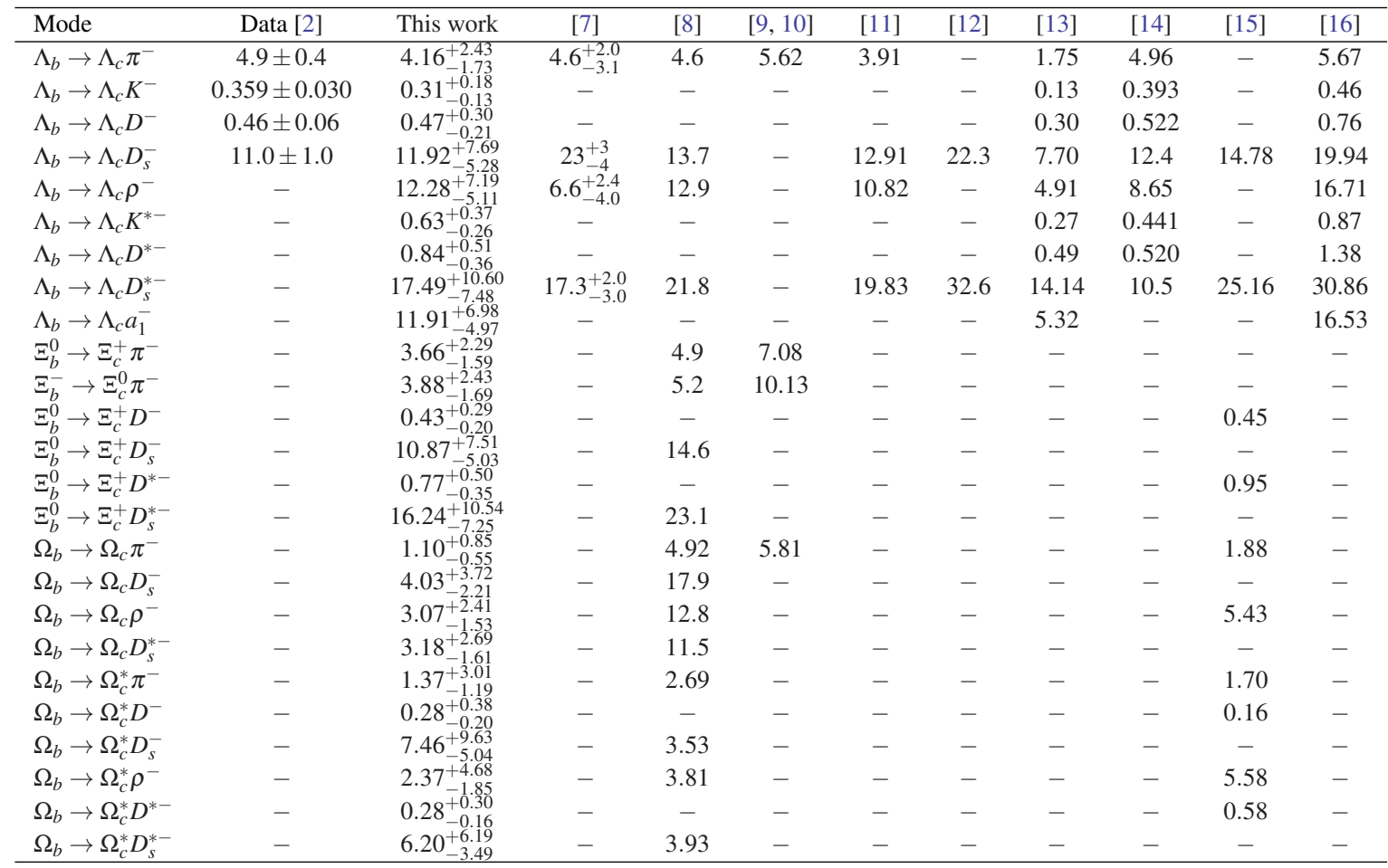

$\Omega_{b} \rightarrow \Omega_{c}(3090) M$ decays can be positive (see Tables 3 and 4). These features are also supported by other works (see Table 6 ).

It will be interesting to comparing $\Lambda_{b} \rightarrow \Lambda_{c}$ (2940)M decays with two different assignments of the configurations of $\Lambda_{c}(2940)$, a radial excite $p$-wave spin-1/2 or spin-3/2 particle. From Tables 1 and 2, we have $\operatorname{Br}\left[\Lambda_{b} \rightarrow \Lambda_{c}\left(2940,3 / 2^{-}\right) P\right] \simeq(1.5 \sim 2) \times \operatorname{Br}\left[\Lambda_{b} \rightarrow \Lambda_{c}\left(2940,1 / 2^{-}\right) P\right]$, $\operatorname{Br}\left[\Lambda_{b} \rightarrow \Lambda_{c}\left(2940,3 / 2^{-}\right) V\right] \simeq \operatorname{Br}\left[\Lambda_{b} \rightarrow \Lambda_{c}\left(2940,1 / 2^{-}\right) V\right]$ and $B r\left[\Lambda_{b} \rightarrow \Lambda_{c}\left(2940,3 / 2^{-}\right) A\right] \simeq$ $\operatorname{Br}\left[\Lambda_{b} \rightarrow \Lambda_{c}\left(2940,1 / 2^{-}\right) A\right]$. The enhancements of the rates of the $3 / 2$ modes compared to those of the $1 / 2$ modes can be traced to the kinematics factor of $m_{\Lambda_{b}}^{2} / m_{\Lambda_{c}(2940)}^{2}$. The asymmetries in $\Lambda_{b} \rightarrow \Lambda_{c}\left(2940,1 / 2^{-}\right) M$ and $\Lambda_{b} \rightarrow \Lambda_{c}\left(2940,3 / 2^{-}\right) M$ decays are similar in most cases, but have larger deviations in the cases of heavy vector mesons. In $\Lambda_{b} \rightarrow \Lambda_{c}(2940) D^{*-}$ and $\Lambda_{c}(2940) D_{s}^{*-}$ decays, the predictions based on the spin- $1 / 2$ configuration, give $-25 \%$ and $-19 \%$, respectively, while the ones based on the spin-3/2 configurations are $-48 \%$ and $-45 \%$, respectively.

The predictions on rates and asymmetries presented in Tables 1, 2, 3 and 4 can be verified experimentally. These information may shed light on the quantum numbers of $\Lambda_{c}(2765), \Lambda_{c}(2940)$ $\Omega_{c}(3050), \Omega_{c}(3090)$ and $\Omega_{c}(3120)$.

This work was supported in part by the Ministry of Science and Technology of R.O.C. under Grant No. 106-2112-M-033-004-MY3. 
Table 6: Various theoretical results on the up-down asymmetries ( $\alpha$ in the unit of $\%$ ) of $\Lambda_{b} \rightarrow \Lambda_{c} M$, $\Xi_{b} \rightarrow \Xi_{c} M, \Omega_{b} \rightarrow \Omega_{c} M$ and $\Omega_{b} \rightarrow \Omega_{c}(2770) M$ decays are compared.

\begin{tabular}{lccccccccc}
\hline Mode & This work & {$[7]$} & {$[8]$} & {$[9,10]$} & {$[12]$} & {$[13]$} & {$[14]$} & {$[15]$} & {$[16]$} \\
\hline$\Lambda_{b} \rightarrow \Lambda_{c} \pi^{-}$ & $-99.99_{-0.00}^{+4.70}$ & -100 & -99 & -99 & - & -99.9 & -99.8 & - & -100 \\
$\Lambda_{b} \rightarrow \Lambda_{c} K^{-}$ & $-99.97_{-0.01}^{+5.02}$ & - & - & - & - & -100 & -100 & - & -100 \\
$\Lambda_{b} \rightarrow \Lambda_{c} D^{-}$ & $-99.45_{-0.55}^{+9.94}$ & - & - & - & - & -98.7 & -99.9 & -98.9 & -98.3 \\
$\Lambda_{b} \rightarrow \Lambda_{c} D_{s}^{-}$ & $-99.19_{-0.81}^{+8.59}$ & -99.1 & -99 & - & -98 & -98.4 & -100 & -98.6 & -97.8 \\
$\Lambda_{b} \rightarrow \Lambda_{c} \rho^{-}$ & $-86.96_{-0.87}^{+5.60}$ & -90.3 & -88 & - & - & -89.8 & -88.8 & - & -87.5 \\
$\Lambda_{b} \rightarrow \Lambda_{c} K^{*-}$ & $-82.96_{-1.11}^{+6.02}$ & - & - & - & - & -86.5 & -85.9 & - & -83.6 \\
$\Lambda_{b} \rightarrow \Lambda_{c} D^{*-}$ & $-36.85_{-4.88}^{+7.08}$ & - & - & - & - & -45.9 & -47.8 & - & -37.1 \\
$\Lambda_{b} \rightarrow \Lambda_{c} D_{s}^{*-}$ & $-32.69_{-5.05}^{+6.82}$ & -43.7 & -36 & - & -40 & -41.9 & -43.9 & -36.4 & -32.7 \\
$\Lambda_{b} \rightarrow \Lambda_{c} a_{1}^{-}$ & $-70.00_{-2.30}^{+7.00}$ & - & - & - & - & -75.8 & - & - & -70.9 \\
$\Xi_{b}^{0} \rightarrow \Xi_{c}^{+} \pi^{-}$ & $-99.98_{-0.00}^{+5.73}$ & - & -100 & -100 & - & - & - & - & - \\
$\Xi_{b}^{-} \rightarrow \Xi_{c}^{0} \pi^{-}$ & $-99.98_{-0.00}^{+5.73}$ & - & -100 & -97 & - & - & - & - & - \\
$\Xi_{b}^{0} \rightarrow \Xi_{c}^{+} D_{s}^{-}$ & $-98.99_{-1.01}^{+10.34}$ & - & -99 & - & - & - & - & - & - \\
$\Xi_{b}^{0} \rightarrow \Xi_{c}^{-} D_{s}^{*-}$ & $-31.84_{-5.72}^{+7.78}$ & - & -36 & - & - & - & - & - & - \\
$\Omega_{b} \rightarrow \Omega_{c} \pi^{-}$ & $59.94_{-18.76}^{+21.34}$ & - & 51 & 60 & - & - & - & - & - \\
$\Omega_{b} \rightarrow \Omega_{c} D_{s}^{-}$ & $55.16_{-19.18}^{+23.98}$ & - & 42 & - & - & - & - & - & - \\
$\Omega_{b} \rightarrow \Omega_{c} \rho^{-}$ & $61.63_{-19.83}^{+22.03}$ & - & 53 & - & - & - & - & - & - \\
$\Omega_{b} \rightarrow \Omega_{c} D_{s}^{*-}$ & $73.53_{-26.54}^{+21.54}$ & - & 64 & - & - & - & - & - & - \\
$\Omega_{b} \rightarrow \Omega_{c}(2770) \pi^{-}$ & $2.60_{-102.23}^{+97.40}$ & - & -38 & - & - & - & - & - & - \\
$\Omega_{b} \rightarrow \Omega_{c}(2770) D_{s}^{-}$ & $-11.70_{-55.10}^{+50.63}$ & - & -22 & - & - & - & - & - & - \\
$\Omega_{b} \rightarrow \Omega_{c}(2770) \rho^{-}$ & $-5.22_{-8899}^{+8.23}$ & - & -75 & - & - & - & - & - & - \\
$\Omega_{b} \rightarrow \Omega_{c}(2770) D_{s}^{*-}$ & $-22.62_{-48.63}^{+42.82}$ & - & -31 & - & - & - & - & - & - \\
\hline
\end{tabular}

\section{References}

[1] R. Aaij et al. [LHCb], JHEP 1705, 030 (2017); Phys. Rev. Lett. 118, no. 18, 182001 (2017).

[2] M. Tanabashi et al. [Particle Data Group], Phys. Rev. D 98, no. 3, 030001 (2018); C.G. Wohl, reviews on "Charmed Baryons", ibid.

[3] H. Y. Cheng and C. W. Chiang, Phys. Rev. D 95, no. 9, 094018 (2017).

[4] R. Aaij et al. [LHCb], JHEP 1408, 143 (2014); JHEP 1404, 087 (2014); Phys. Rev. Lett. 112, 202001 (2014).

[5] C. K. Chua, Phys. Rev. D 99, no. 1, 014023 (2019).

[6] C. K. Chua, Phys. Rev. D 100, no. 3, 034025 (2019).

[7] T. Mannel and W. Roberts, Z. Phys. C 59, 179 (1993).

[8] H. Y. Cheng, Phys. Rev. D 56, 2799 (1997) Erratum: [Phys. Rev. D 99, no. 7, 079901 (2019)].

[9] M. A. Ivanov, J. G. Korner, V. E. Lyubovitskij and A. G. Rusetsky, Mod. Phys. Lett. A 13, 181 (1998).

[10] M. A. Ivanov, J. G. Korner, V. E. Lyubovitskij and A. G. Rusetsky, Phys. Rev. D 57, 5632 (1998).

[11] A. K. Giri, L. Maharana and R. Mohanta, Mod. Phys. Lett. A 13, 23 (1998).

[12] Fayyazuddin and Riazuddin, Phys. Rev. D 58, 014016 (1998).

[13] R. Mohanta et al., Prog. Theor. Phys. 101, 959 (1999.)

[14] J. Zhu, Z. T. Wei and H. W. Ke, Phys. Rev. D 99, no. 5, 054020 (2019).

[15] T. Gutsche, M. A. Ivanov, J. G. Körner and V. E. Lyubovitskij, Phys. Rev. D 98, no. 7, 074011 (2018).

[16] H. W. Ke, N. Hao and X. Q. Li, Eur. Phys. J. C 79, no. 6, 540 (2019). 